\title{
Understanding kangaroo care and its benefits to preterm infants
}

This article was published in the following Dove Press journal:

Pediatric Health, Medicine and Therapeutics

18 March 2015

Number of times this article has been viewed

\author{
Marsha L Campbell-Yeo ${ }^{1-4}$ \\ Timothy C Disher' \\ Britney L Benoit ${ }^{\prime}$ \\ C Celeste Johnston ${ }^{2,4,5}$
}

'School of Nursing, Dalhousie University, ${ }^{2}$ Department of Pediatrics, IWK Health Centre, ${ }^{3}$ Department of Psychology and Neuroscience, Dalhousie University, ${ }^{4}$ Centre for Pediatric Pain Research, IWK Health Centre, Halifax, NS, Ingram School of Nursing, McGill University, Montréal, QC, Canada
Correspondence: Marsha L Campbell-Yeo School of Nursing, Faculty of Health Professions, 5869 University Avenue, PO Box 15000, Halifax, NS B3H 4R2, Canada

$\mathrm{Tel}+\mathrm{I} 9024944283$

Fax + I 9024943487

Email marsha.campbell-yeo@dal.ca

\begin{abstract}
The holding of an infant with ventral skin-to-skin contact typically in an upright position with the swaddled infant on the chest of the parent, is commonly referred to as kangaroo care $(\mathrm{KC})$, due to its simulation of marsupial care. It is recommended that $\mathrm{KC}$, as a feasible, natural, and cost-effective intervention, should be standard of care in the delivery of quality health care for all infants, regardless of geographic location or economic status. Numerous benefits of its use have been reported related to mortality, physiological (thermoregulation, cardiorespiratory stability), behavioral (sleep, breastfeeding duration, and degree of exclusivity) domains, as an effective therapy to relieve procedural pain, and improved neurodevelopment. Yet despite these recommendations and a lack of negative research findings, adoption of $\mathrm{KC}$ as a routine clinical practice remains variable and underutilized. Furthermore, uncertainty remains as to whether continuous $\mathrm{KC}$ should be recommended in all settings or if there is a critical period of initiation, dose, or duration that is optimal. This review synthesizes current knowledge about the benefits of $\mathrm{KC}$ for infants born preterm, highlighting differences and similarities across low and higher resource countries and in a non-pain and pain context. Additionally, implementation considerations and unanswered questions for future research are addressed.
\end{abstract}

Keywords: kangaroo care, skin-to-skin contact, infant, preterm, review

\section{Introduction}

Mothers hold babies to their chest instinctively. Currently, in less well developed societies, where cribs, strollers, and infant seats are not common, mothers or other caregivers carry their infants on their chest for many hours a day. ${ }^{1}$ Often there is nothing between the caregiver's chest and the baby's skin other than a diaper. This paradigm of holding an infant with ventral skin-to-skin contact (SSC), typically in an upright position and with the swaddled infant on the chest of the parent, is also commonly referred to as kangaroo care $(\mathrm{KC})$, due to its simulation of marsupial care. While there are no recordings of infant care from the distant past, it is likely that $\mathrm{KC}$ of newborns has been practiced for eons. An example of the basic survival value of skin-to skin contact between infant and mother can be demonstrated when there is no interference from health care providers at delivery, specifically when the infant is placed skin-to-skin on its mothers chest at birth, within 20 minutes it will work its way toward the nipple and suckle. ${ }^{2}$

The medical use of this natural phenomenon was originally introduced by Edgar Rey Sanabria in Columbia in 1978 as a strategy to replace the function of incubators, which were in short supply in that country. Infants who were preterm, but otherwise stable, were put in continuous $\mathrm{KC}$ with their mothers. There are variations in $\mathrm{KC}$ practices, but all of it involves SSC. For example, kangaroo mother care (KMC) 
refers to SSC that is provided continuously until the infant begins to sweat and resist the position, an indication of more mature temperature regulation and development. Breastfeeding is exclusive, and discharge home occurs earlier than usual, when the baby is stable and the mother is comfortable providing continuous SSC. ${ }^{3-5}$ Fathers and other family members can also be providers when the mother is unavailable. ${ }^{6}$

In resource-rich countries, $\mathrm{SSC}$ is seen as complementary to incubator care, and so continuous $\mathrm{KC}$ is rare. Implementation of SSC in hospitals has largely been motivated by a desire to humanize what has become a medical experience, and as partial fulfillment of the requirements set out in the Baby Friendly Hospital Initiative (BFHI). ${ }^{7}$ The purpose of SSC in resource-rich countries has therefore been focused on facilitating infant transition to extrauterine life, promoting early bonding and establishing exclusive breastfeeding. SSC has shown added benefit for the mother, including reduced incidence of post-partum hemorrhage; ${ }^{8}$ however, this review focuses primarily on the benefits to infants. More recently, strong evidence related to the pain-relieving benefits of SSC, almost exclusively studied in developed countries, has emerged.

$\mathrm{KC}$ has been studied for its effect on mortality, morbidity, physiological stability, breastfeeding, parental bonding, development, and pain control. ${ }^{9-11}$ Yet despite consistent positive findings for all outcomes, adoption of $\mathrm{KC}$ as a routine clinical practice remains extremely variable across settings. It is recommended that $\mathrm{KC}$ is a feasible, natural, and costeffective intervention, and should be standard of care in the delivery of quality health care for all infants, regardless of geographic location or economic status. What remains uncertain is whether continuous $\mathrm{KC}$ should be recommended in all settings or if there is a critical period of initiation, dose, or duration that is optimal. This review provides a synthesis of our current knowledge about the benefits of $\mathrm{KC}$, highlighting differences and similarities across gestational age, low and higher resource countries, and in a non-pain and pain context. Additionally, implementation considerations and unanswered questions for future research are addressed.

\section{Benefits of KC}

Since the inception of $\mathrm{KC}$ as a low-cost alternative to incubator care in areas with limited resources, clinicians and researchers have, over time, documented both physiologic and behavioral benefits for infant and mother (see Table 1). Many of these benefits have been researched sufficiently to permit meta-analysis, such that two Cochrane reviews exist on the subject. ${ }^{9,10}$ One focuses on healthy term or latepreterm newborns, ${ }^{9}$ while the second includes low birth weight infants. ${ }^{10}$

\section{Physiologic benefits Homeostasis (temperature regulation, physiological stability, blood glucose)}

When compared with standard care (incubator, radiant warmer, or open crib), $\mathrm{KC}$ has shown benefits for homeostasis. Preterm infants who receive $\mathrm{KC}$ are more likely to maintain a healthy body temperature, and show increased cardiorespiratory stability. ${ }^{10,12-20}$ Looking at the entire hospitalization, $\mathrm{KC}$ is associated with decreased likelihood of infection, severe illness, and death. ${ }^{10,21}$ Additional evidence for the positive influence of $\mathrm{KC}$ exposure on autonomic regulation comes from a recent longitudinal study, ${ }^{22}$ which showed a significant increase in baseline autonomic stability at 10 years follow-up. Even a very small amount of KC ( 1 hour a day for 14 days) provided to preterm infants compared with infants cared for only in an incubator was associated with improved infant and maternal outcomes. These findings are of particular interest because they are the first to demonstrate the long-lasting value of early $\mathrm{KC}$.

\section{Implications for practice}

The evidence for the ability of $\mathrm{KC}$ to promote homeostasis is strong, especially in developing countries, where good evidence suggests that continuous $\mathrm{KC}$ can reduce mortality. ${ }^{10}$ Unfortunately, the clinical picture is less clear in the developed world. The best evidence available is focused on intermittent use of $\mathrm{KC}$, and while homeostatic benefits should theoretically persist when $\mathrm{KC}$ is continuous, there is a lack of studies designed to address this. Combined analysis conducted by Conde-Agudelo et al found that benefits that were clear in less developed countries (eg, reduction in sepsis, mortality, and severe illness) were not present when studies were limited to those in developed countries. ${ }^{10}$ There remain limitations in making conclusions regarding the optimal time spent in $\mathrm{KC}$ to achieve maximum benefits. Feldman et $\mathrm{al}^{22}$ offer compelling evidence that an average of 1 hour a day of $\mathrm{KC}$ may impart long-lasting benefits, but evidence is still lacking as to whether shorter or longer time periods may impart different benefits. Clinicians should use best judgment in balancing the illness acuity of individual infants, parent availability, and potential benefits.

\section{Implications for research}

Benefits that were discovered through research in developing countries should not be extrapolated to better resourced 


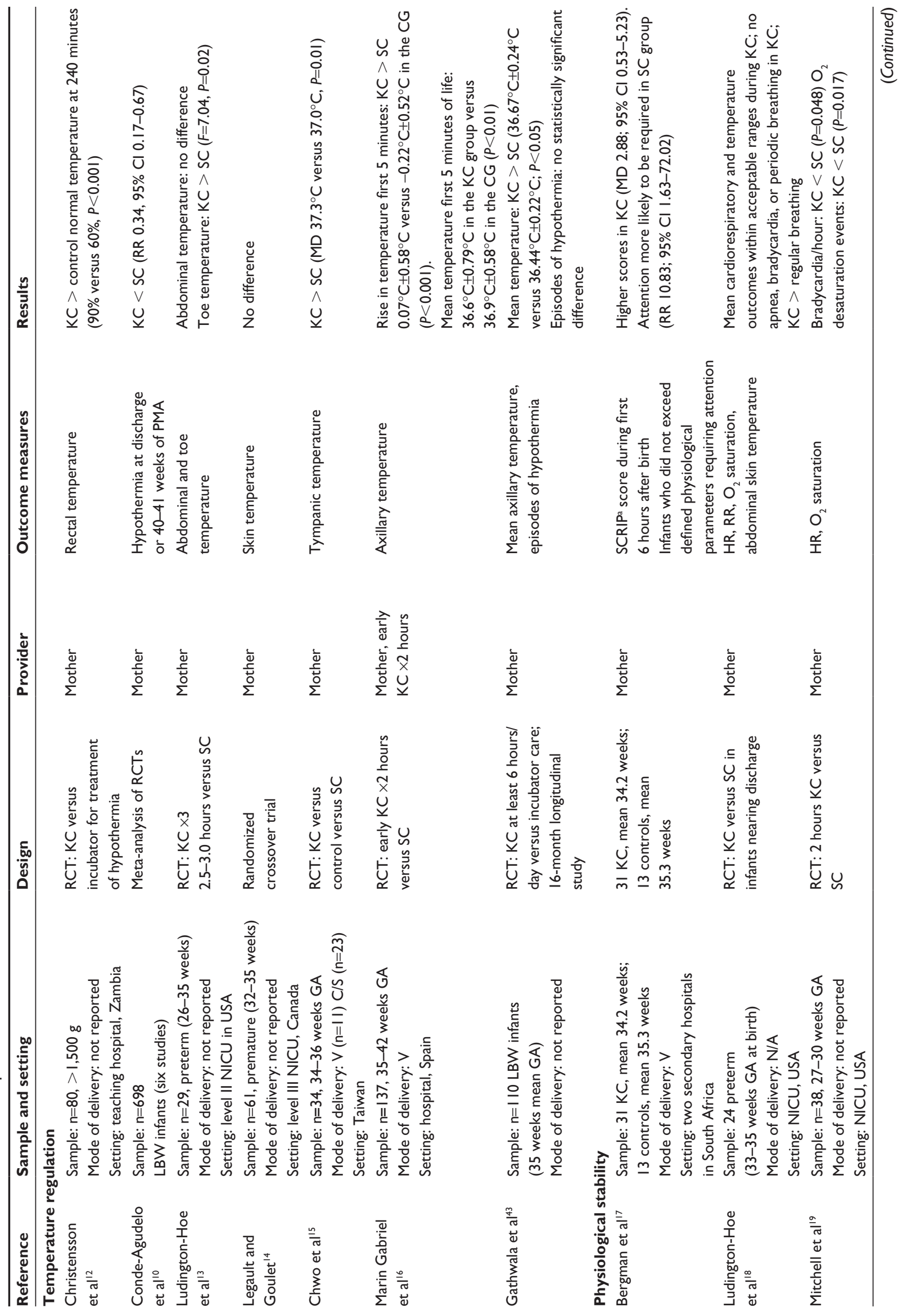




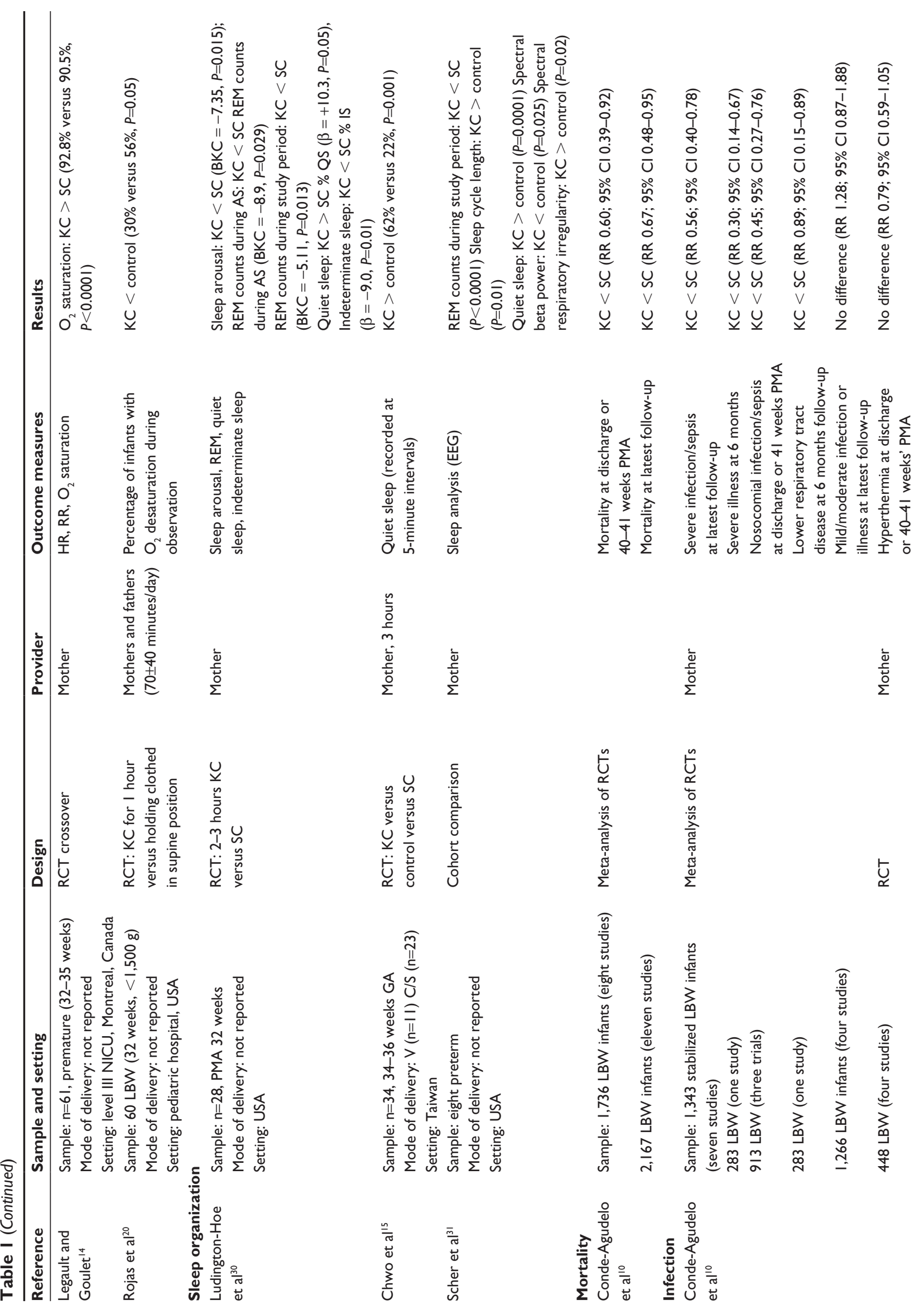



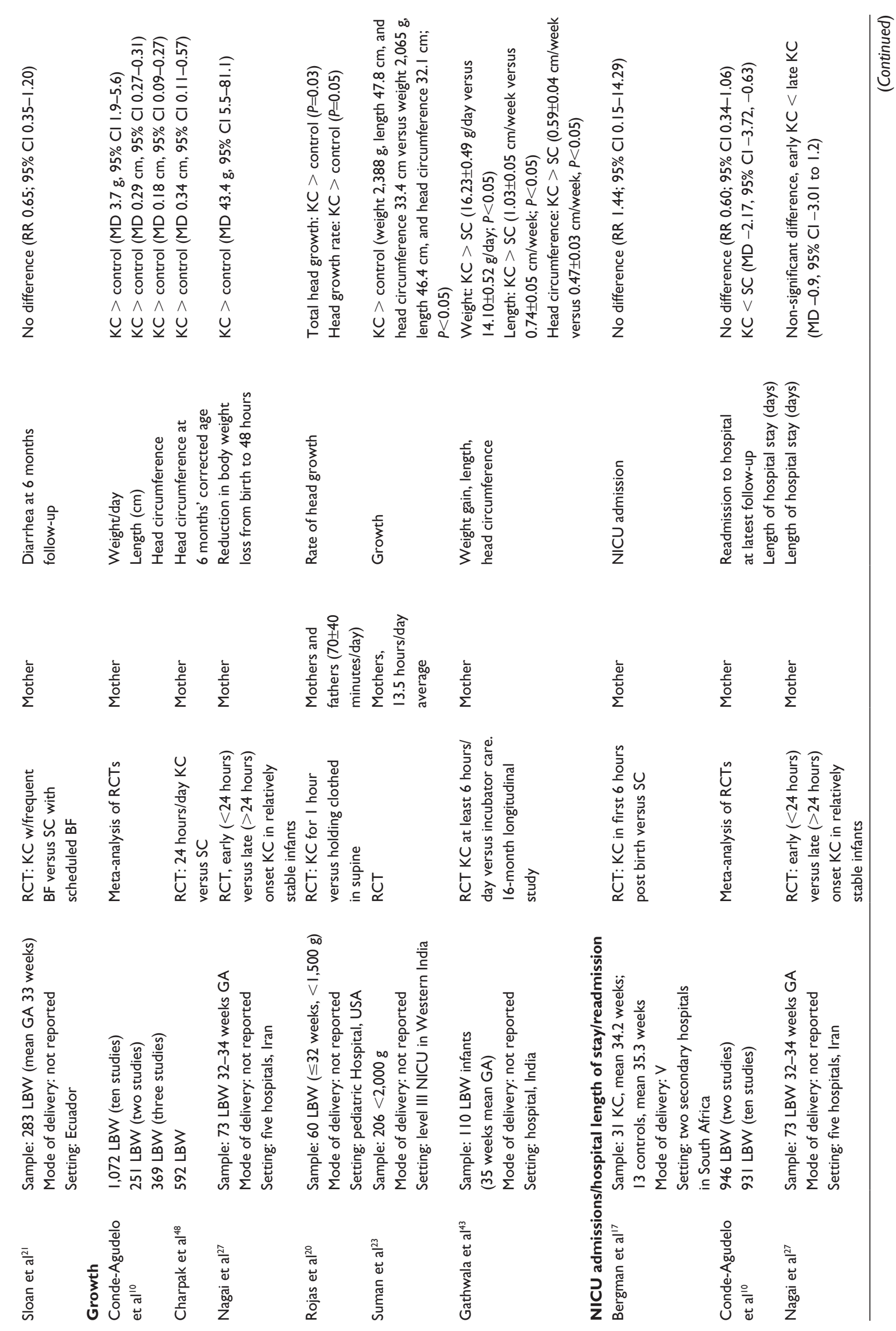


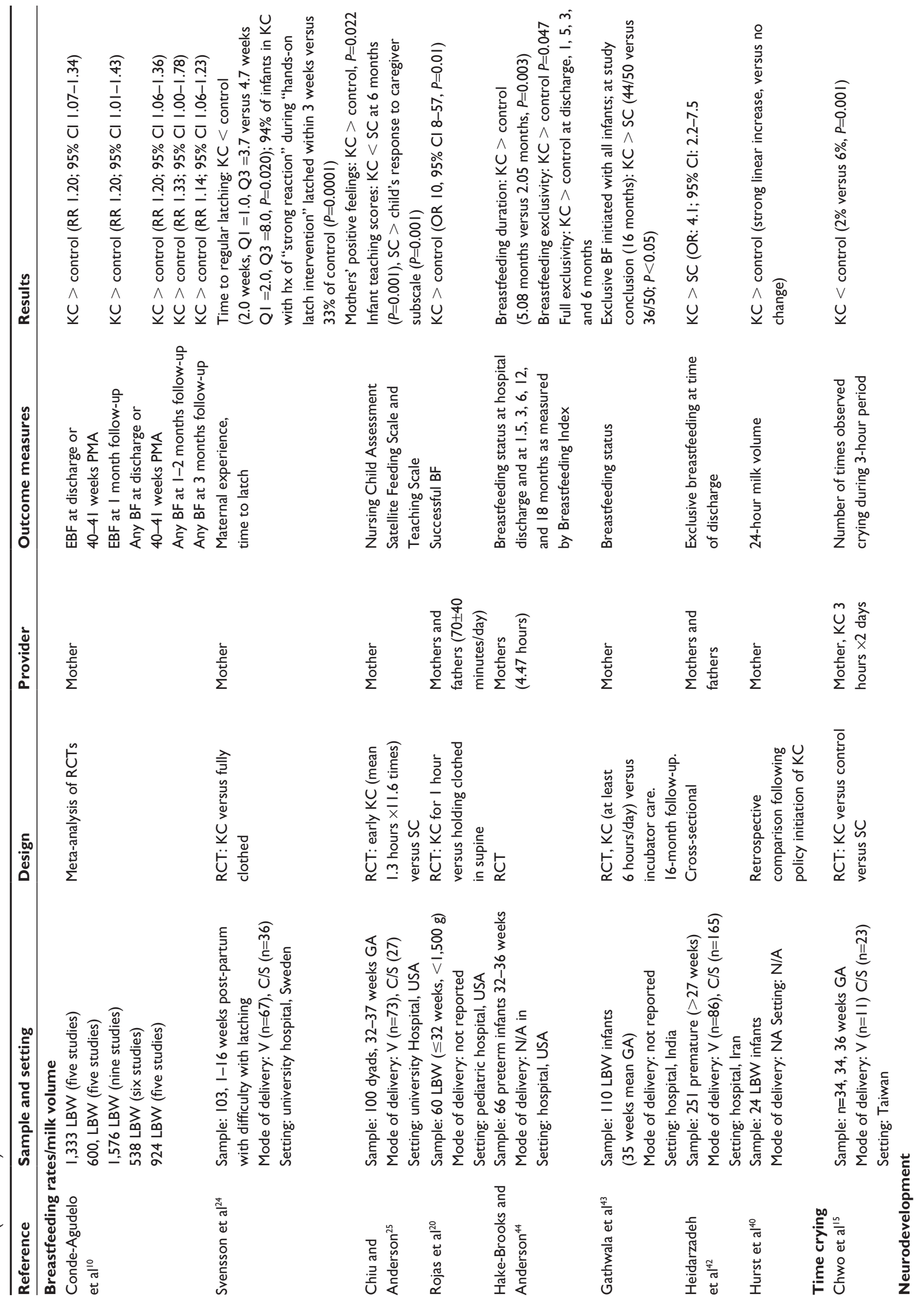



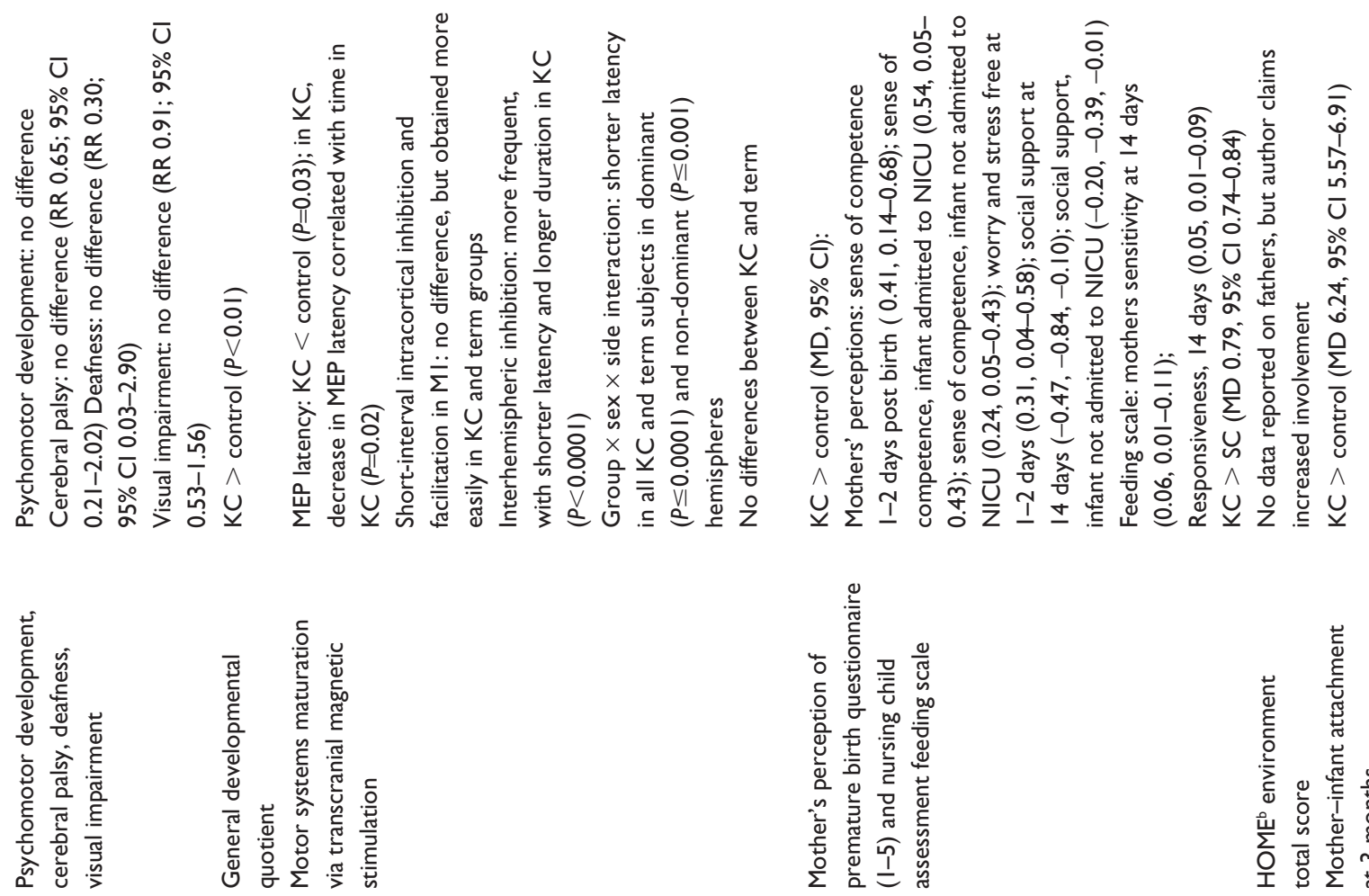

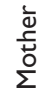

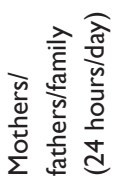

$\frac{\grave{\Phi}}{\frac{\grave{L}}{\Sigma}}$
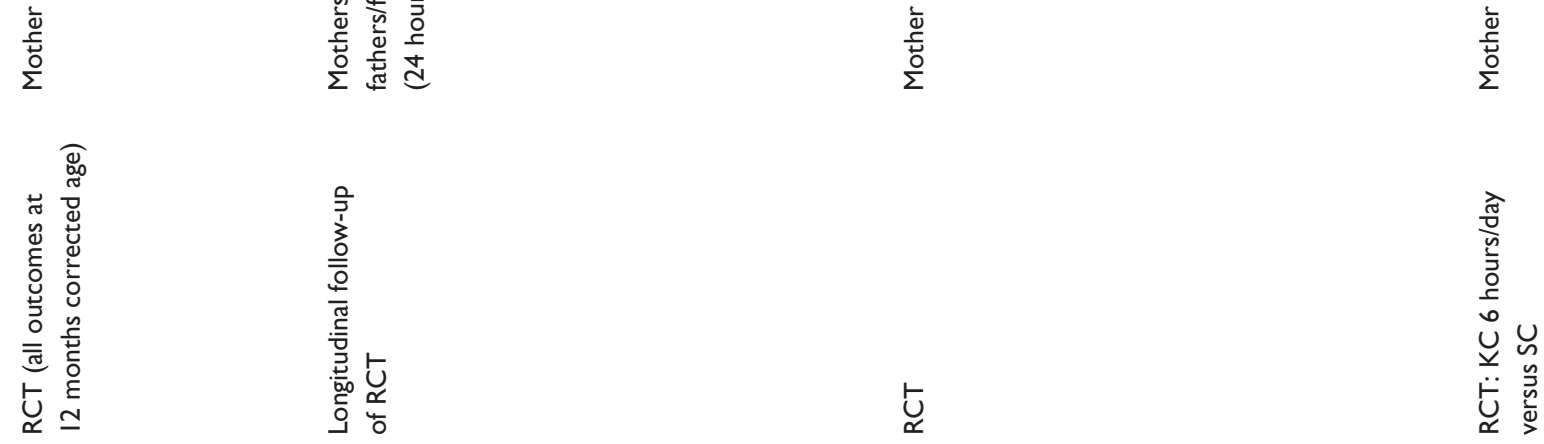

$\stackrel{\leftarrow}{\longleftarrow}$

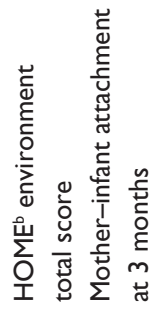

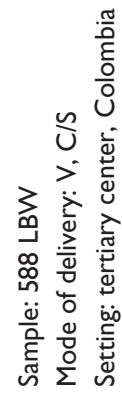
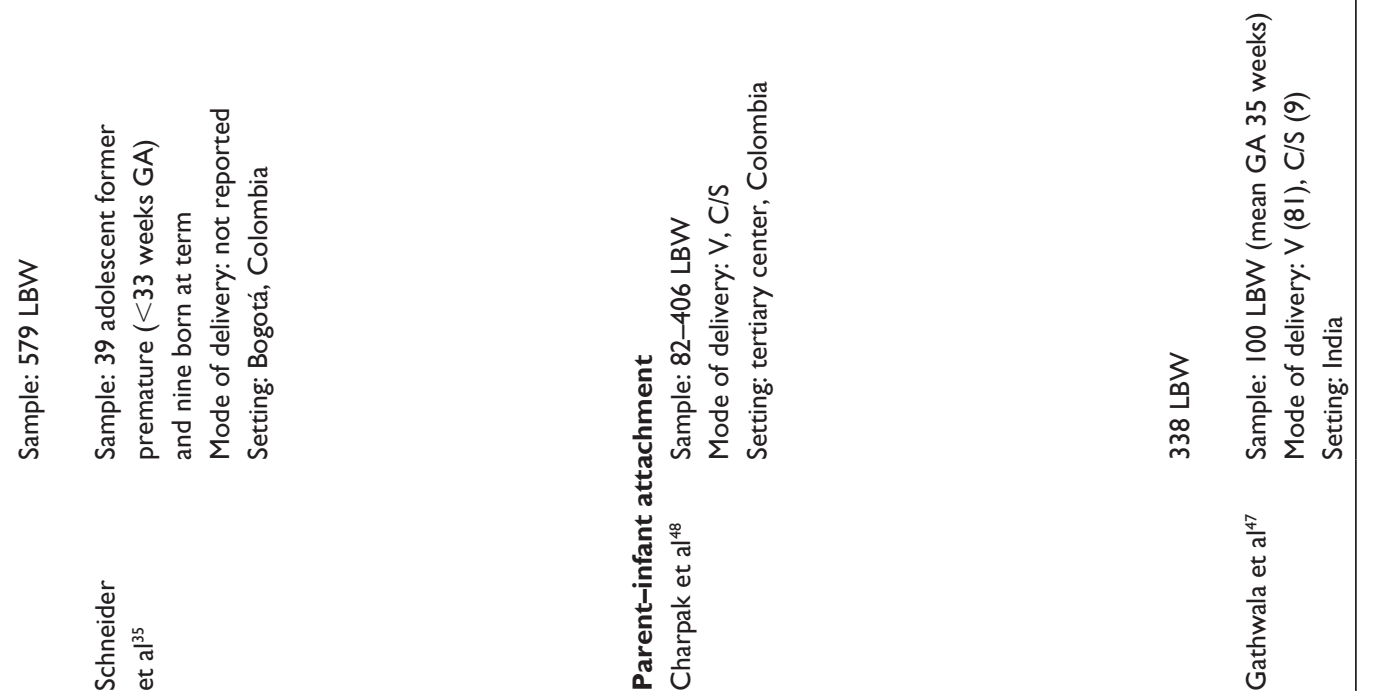


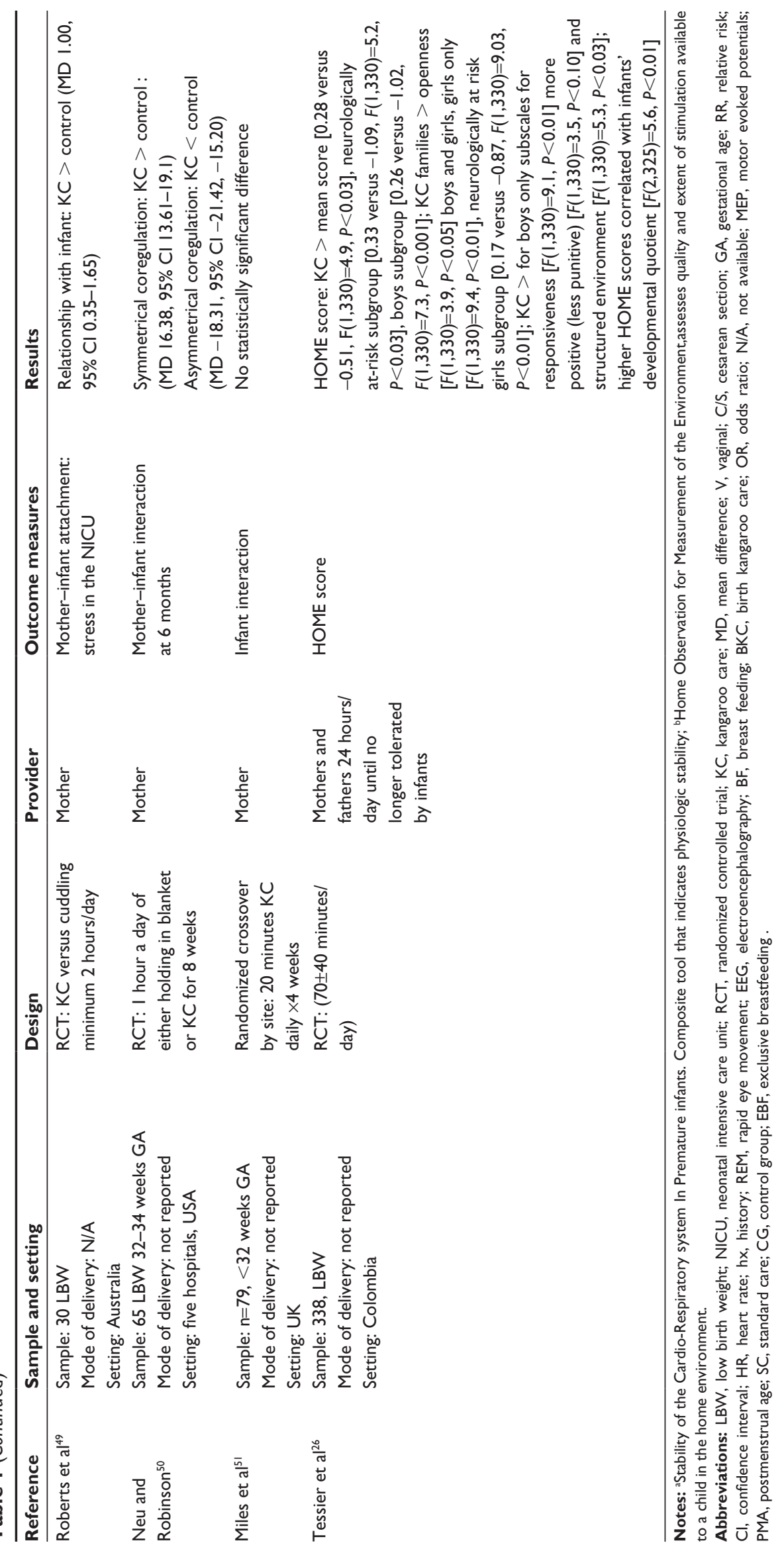


countries. There remains a paucity of studies investigating the effect of $\mathrm{KC}$ on mortality, infection, and serious illness in resource-rich countries. Methodologically rigorous studies are needed to understand how to maximize the clinical benefits of $\mathrm{KC}$ in hospital environments where advanced support is readily available. Many of the questions that remain are most appropriately answered by randomized controlled trials. In both developed and developing countries, our understanding of the physiological benefits of $\mathrm{KC}$ would benefit from routine use of composite physiological measures from which investigators can interpret a more meaningful clinical picture. In a review of the benefits of $\mathrm{KC}$ in term children, Moore suggests the use of SCRIP scores, (eg, stability of the cardiorespiratory system as the primary physiological outcome). ${ }^{9}$ This would help draw conclusions that more broadly address stability as opposed to attempting to interpret outcomes separately.

There is little evidence from which to determine minimum times for $\mathrm{KC}$ to maximize physiological benefits, which are of particular interest to clinicians in countries like Canada and the United States of America (USA) where parent availability is often limited. Continuous KC potentially represents a free alternative to expensive equipment, but the price may be high for parents who bear the burden of additional costs associated with being available in neonatal intensive care units. A recent systematic review and meta-analysis ${ }^{28}$ of 29 papers addressing parental experiences during $\mathrm{KC}$, including 401 mothers and 94 fathers, revealed two overarching themes, ie, a beneficial and restoring effect on both themselves and their child(ren), but also an increased burden, which was a draining experience. Improving our understanding of the optimum daily duration of $\mathrm{KC}$ will be important in order to ensure that we do not unjustifiably transfer burden from the health care system to parents.

Late-preterm infants, primarily delivered in developed countries, who are deemed healthy enough to remain on the post partum ward, have been excluded from much of the current literature. ${ }^{9}$ If physiological stability can be improved by introduction of early and sustained $\mathrm{KC}$ interventions for this population, there is potential for considerable impact on health care costs, family burden, and reduction of interventions. Similarly, there is a lack of analysis of benefits for infants born via cesarean section. In the studies included in this review, none considered analysis of outcomes in cesarean deliveries separately. This lack of evidence was one of the issues highlighted in a recent Cochrane review of vaginal versus cesarean birth for preterm infants. ${ }^{29}$ While results of that meta-analysis found no difference in any of the reported outcomes (respiratory distress, Apgar scores, mechanical ventilation, supplemental oxygen), investigators were only able to include four trials, the most recent published in 1996.

For preterm or low birth weight infants, there is a need for more research examining the effectiveness of continuous early $\mathrm{KC}$ for both nonstabilized and relatively stabilized infants..$^{10}$ Perhaps unstable infants would benefit differentially from $\mathrm{KC}$ when compared with stable infants. Moreover, it remains unclear whether physiological benefits are variable depending on the $\mathrm{KC}$ provider, because there is so little research comparing mothers and alternative providers, such as fathers, grandparents, or trained volunteers. ${ }^{22}$

\section{Growth, neurodevelopment, and neurosensory impairment}

Perhaps one of the most interesting benefits of $\mathrm{KC}$ is the effect on sleep, neurodevelopment, and growth. A recent study by Feldman et a ${ }^{22}$ provides compelling evidence in this domain as well as in the physiological domain. They found that infants who received an average of 1 hour of $\mathrm{KC}$ for 14 days showed a more organized sleep-wake cycle at 10 years of age. Short-term benefits of KC on sleep patterns in preterm infants have been well established and include an increase in quiet sleep, longer cycles, and increased respiratory regularity. ${ }^{15,30,31}$

As a consequence of the tremendous rate of neurodevelopment that occurs in utero, it is no surprise that preterm infants often suffer neurophysiological sequelae. ${ }^{32,33}$ Two recent cohort studies used electroencephalographic complexity to measure differences in neurological maturity between preterm infants who received $\mathrm{KC}$ and those who did not. While sample sizes were small, the investigators were able to identify a relationship between $\mathrm{KC}$ and increased electroencephalographic complexity. ${ }^{34}$ Additionally, the results showed an increase in primary motor cortex synchronization in response to transcranial magnetic stimulation in the group of infants that received KC. ${ }^{35}$ These results suggest that $\mathrm{KC}$ plays a role in supporting neurodevelopment, which is consistent with earlier findings. ${ }^{31}$

A recent clinical trial in an Indian hospital compared sustained $\mathrm{KC}$ with conventional care in preterm infants. The infants were enrolled in groups of five, with the smallest three infants in each group being assigned to the $\mathrm{KC}$ condition. ${ }^{36}$ Growth at a corrected gestational age of 40 weeks was similar between the intervention group and the control group, but infants receiving $\mathrm{KC}$ achieved more rapid physical growth after this point. Evidence for an association between $\mathrm{KC}$ and 
augmented growth comes from the meta-analysis by CondeAgudelo et al, which found benefits for both continuous and intermittent $\mathrm{KC} .{ }^{10}$ When $\mathrm{KC}$ was continuous (KC) it was associated with an increase in weight gain, length, and head circumference.

\section{Clinical implications}

Current evidence suggests that $\mathrm{KC}$ improves sleep, neurodevelopment, and growth, and should therefore be encouraged in clinical practice. While there is a lack of guidance regarding the optimal duration, the compelling outcomes described by Feldman et al were the result of just 14 days of sessions lasting an average of 1 hour. ${ }^{20}$ Given the lack of uptake of any variation of $\mathrm{KC}$ in some practice settings, it would be reasonable to recommend that all infants should have $\mathrm{KC}$ initiated as soon as possible after birth and receive at least this minimal dose daily.

\section{Research implications}

The potential for $\mathrm{KC}$ to impact neurodevelopment and growth in preterm infants is exciting, but there is still much left to be understood. Dose-response studies would be particularly interesting because they would help families and clinicians to collaborate in order to achieve the best outcomes for the least cost. Studies should be randomized when possible in order to help control for unknown confounding variables.

Investigators should take advantage of the diverse range of instruments available to them in order to learn more about the neurodevelopment changes associated with $\mathrm{KC}$. For example, is the hypothesis that $\mathrm{KC}$ increases the rate of neurodevelopment supported by brain imaging? Are the benefits exclusive to pathways related to attention and sleep regulation, or do preterm infants who receive $\mathrm{KC}$ have more rapid peripheral neurodevelopment as well?

Studies designed to elucidate the mechanisms via which KC imparts its benefits are lacking. Sleep, neurodevelopment, weight, and length are clinically relevant outcomes but we also need studies designed to tease out how $\mathrm{KC}$ interacts with these.

\section{Behavioral benefits Breastfeeding rates}

The BFHI, devised by the World Health Organization, is an international set of guidelines to promote, protect, and support breastfeeding. ${ }^{7}$ Provision of $\mathrm{KC}$ is one of the "ten steps to successful breastfeeding" outlined in the BFHI. Although initially developed for healthy term infants, an international group of experts has made recommendations for adapting the "ten steps" of the BFHI to be applicable to ill and premature infants in neonatal settings. ${ }^{37}$ In the modified BFHI for neonatal units proposed by Nyqvist et al, ${ }^{37}$ provision of early, continuous, and prolonged $\mathrm{KC}$ without unjustified restrictions is cited as crucial to improving breastfeeding outcomes in this vulnerable infant population.

Evidence supports the influence of $\mathrm{KC}$ in increasing maternal milk volume and promoting breastfeeding exclusivity and duration in preterm infants. ${ }^{38-40}$ Flacking et $\mathrm{al}^{41}$ used a prospective longitudinal design to examine the influence of $\mathrm{KC}$ on breastfeeding in two age groups: those born very preterm (less than 32 weeks' gestational age) and preterm (32-36 weeks' gestational age). They found that in very preterm infants, those who were breastfeeding at 1, 2, 5, and 6 months post discharge has significantly more $\mathrm{KC}$ time in hospital. In randomized controlled trials comparing $\mathrm{KC}$ interventions with standard care, preterm infants demonstrate initiation of earlier breastfeeding, ${ }^{10}$ higher breastfeeding exclusivity, ${ }^{10,42}$ and a longer duration of breastfeeding ${ }^{10,43,44}$ when compared with infants who are cared for in an incubator or are wrapped in blankets when held by their mothers.

\section{Clinical implications}

Based on consistent evidence for $\mathrm{KC}$ in promoting breastfeeding, clinicians should encourage $\mathrm{KC}$ for preterm infants both in the neonatal intensive care unit and following hospital discharge ${ }^{5,6}$ Given the variability in duration of $\mathrm{KC}$ provided across studies, there is no consensus regarding the length of time required to optimize the benefit for breastfeeding outcomes. Therefore, it is recommended that mothers be informed of the benefits of $\mathrm{KC}$ for breastfeeding, and that they are encouraged and supported in providing $\mathrm{KC}$ as early as possible (ideally from birth) and for as long and as often as they would like. ${ }^{31}$

\section{Limitations and implications for future research}

A limitation in the research examining $\mathrm{KC}$ and breastfeeding is the reliance on maternal self-report. Given the potential for bias in reporting breastfeeding outcomes due to the social desirability of exclusive breastfeeding, it is important to interpret the findings with caution. While some researchers ${ }^{44}$ have considered and controlled for baseline maternal intentions to breastfeed, this is not consistently done. Given the significant influence of prenatal intentions to breastfeed in predicting longterm breastfeeding outcomes, ${ }^{45}$ this is an important variable to measure and report in future studies. While Flacking et $\mathrm{a}^{41}$ 
found that $\mathrm{KC}$ had the greatest benefit for very preterm infants (born at less than 32 weeks' gestational age), research in this young age group is limited. Future studies examining the relationship between $\mathrm{KC}$ and breastfeeding initiation, duration, and exclusivity in infants born at a gestational age of less than 32 weeks is needed. There was variability in measures of exclusivity of breastfeeding, length of $\mathrm{KC}$ intervention time in clinical trials, follow-up time points, and control interventions used (eg, incubator care, being wrapped in blankets when held) across the literature. Possibilities of both overestimation and underestimation of the effect of $\mathrm{KC}$ on breastfeeding stem from issues such as the treatment of breastfeeding as a dichotomous variable, failure to capture information such as nipple protractility, and "standard care" conditions that include breastfeeding counseling expertise which may not accurately reflect the day to day reality of the unit. ${ }^{9}$ Consistency in interventions and outcomes is necessary to strengthen future research.

\section{Parent-infant attachment}

Attachment is defined as the emotional connection that is formed between infants and caregivers, and it is relevant for clinicians to consider this in infants of all gestational ages, especially in those born preterm. ${ }^{46}$ Infant-maternal relationships have been shown to be less positive in infants born preterm, and evidence suggests that poor attachment can contribute to more negative outcomes. ${ }^{46,47}$

Charpak et $\mathrm{al}^{48}$ found that mothers in a $\mathrm{KC}$ group scored more favorably on sense of competence, feelings of worry or stress, sensitivity, and infant responsiveness. Total attachment scores determined through structured interviews by Gathwala et $\mathrm{al}^{47}$ were higher in the $\mathrm{KC}$ group than in controls. An improved relationship with the infant was supported by higher scores in an investigation by Roberts et al, ${ }^{49}$ and $\mathrm{KC}$ motherinfant pairs showed more symmetrical and less asymmetrical coregulation in a recent study that used the still-face paradigm tool as one of their outcomes. ${ }^{50}$ Feldman et $\mathrm{al}^{22}$ provided additional evidence supporting the relationship between $\mathrm{KC}$ and increased attachment behaviors across the post partum period in addition to greater mother-child reciprocity at 10 years. In one study excluded for using a crossover design, the investigators found no differences between $\mathrm{KC}$ and standard care at 4 or 12 months of age in measurement of infant interaction. ${ }^{51}$

\section{Clinical implications}

A significant barrier to the provision of $\mathrm{KC}$ is the lack of facilities available for parents to stay (ie, sleep and cooking) near the infant to promote prolonged $\mathrm{KC}$. This is a significant issue in both poorly resourced and resourced areas, although the trend toward single room versus open bay neonatal units and $\mathrm{KC}$ centers may lessen this concern and should be supported. ${ }^{52}$

\section{Research implications}

A consistent approach to the measurement of parent-infant attachment would be of benefit because measures vary and make interpretation more difficult. The current evidence suggests that $\mathrm{KC}$ promotes greater parent-infant attachment, and the implications of this benefit should cause investigators to consider the inclusion of measures for attachment. Due to the potential neurodevelopment consequences of poor parent-infant attachment, increased information combined with longitudinal designs may help understand the basis of the seemingly neuroprotective effects of $\mathrm{KC}$. More positive attachment may also partially explain some of the observed benefits to breastfeeding initiation and duration. There remains a knowledge gap with regard whether the same benefits in relationships that are observed between motherinfant dyads as a result of $\mathrm{KC}$ are also observed between father-infant ${ }^{20}$ dyads.

\section{$K C$ in the context of pain}

Given the benefits of improved physiological stability, and enhanced sleep and regulation, investigation of $\mathrm{KC}$ to diminish newborn procedural pain has become a rapidly growing field of study (see Table 2). The role of $\mathrm{KC}$ in this context was first examined in full-term infants in 2000 by Gray et al. ${ }^{53}$ Three years later, Johnston et al examined its effectiveness in preterm infants at a gestational age of 32 to 36 weeks at birth. ${ }^{54}$ Both studies reported a significant lowering of behavioral pain responses, and numerous other studies followed with similar results favoring $\mathrm{KC}$. A recent Cochrane review included 19 randomized trials $(n=1,594$ infants, with 765 being term infants ${ }^{54-67}$ ) and used physiological, behavioral, and composite measures as the primary outcomes. ${ }^{11}$ The majority of the studies ${ }^{53-59,64-70}(n=1,219)$ compared KC with standard care or no treatment, and used heel lance as the painful procedure. . $^{53,54,56-67,71}$

\section{Kangaroo care compared with incubator control Physiological parameters}

Physiological indicators reported were heart rate response, heart rate recovery, heart rate variability, oxygen saturation during the painful procedure, oxygen saturation after the painful procedure, and change in oxygen saturation. Twelve studies examined heart rate or heart rate variability during 


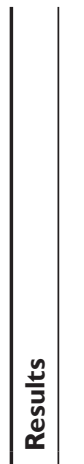

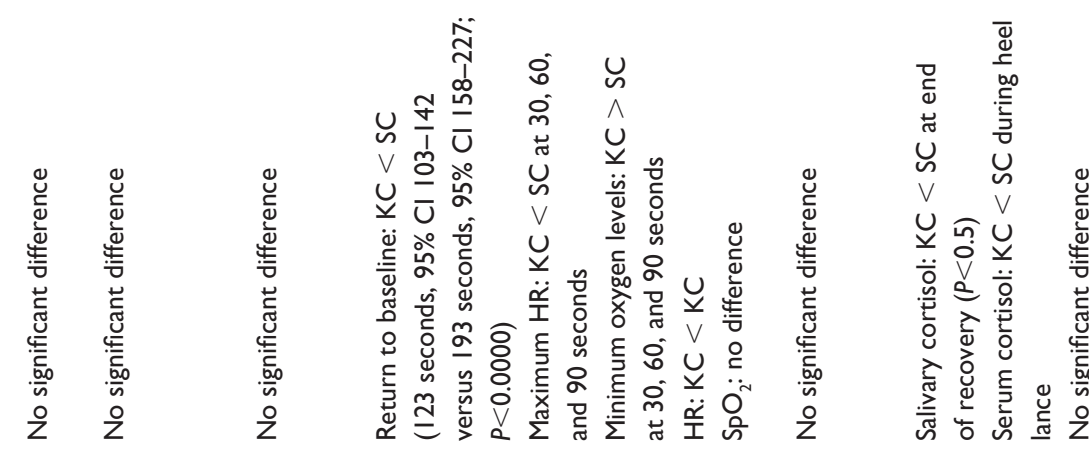

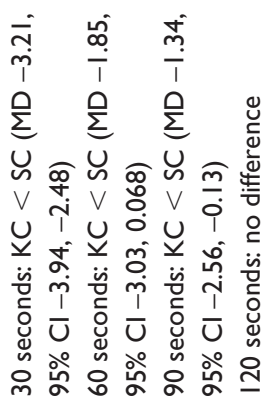

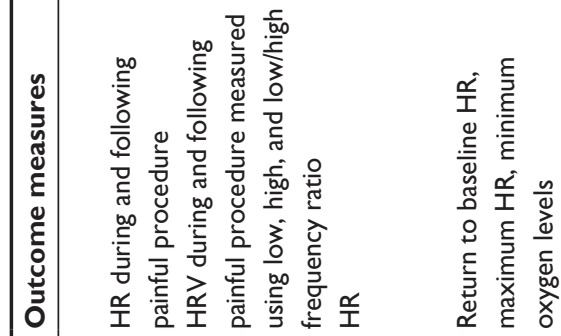

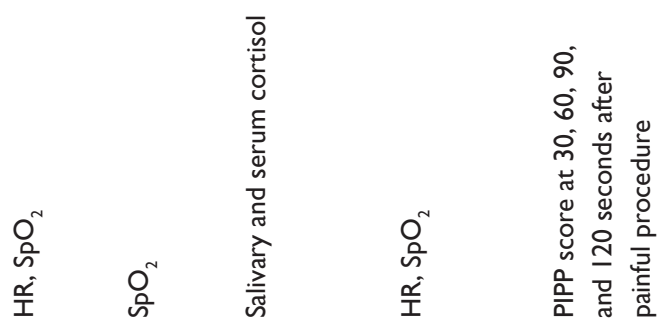

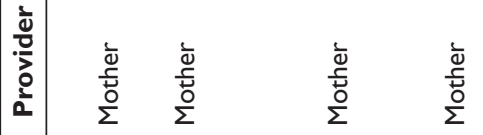

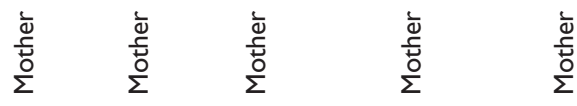

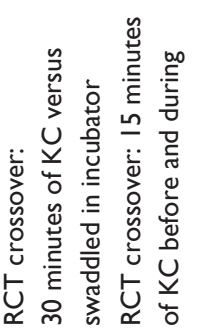

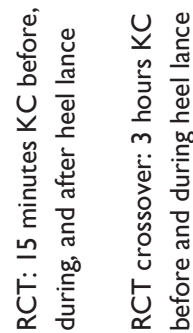
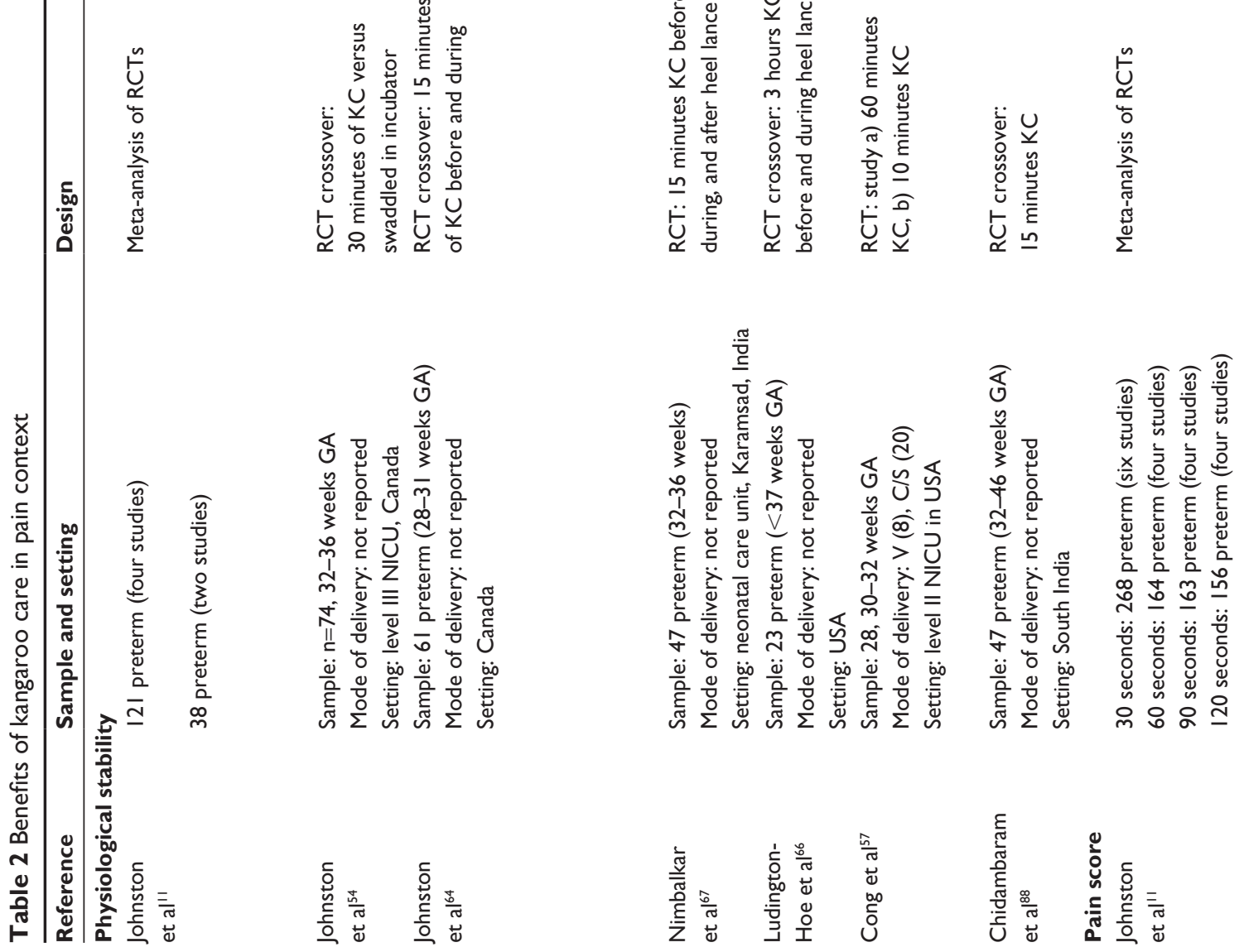

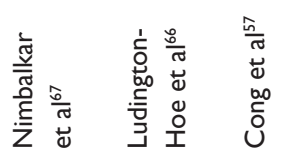

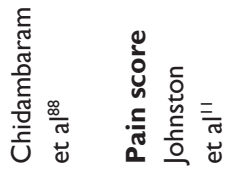



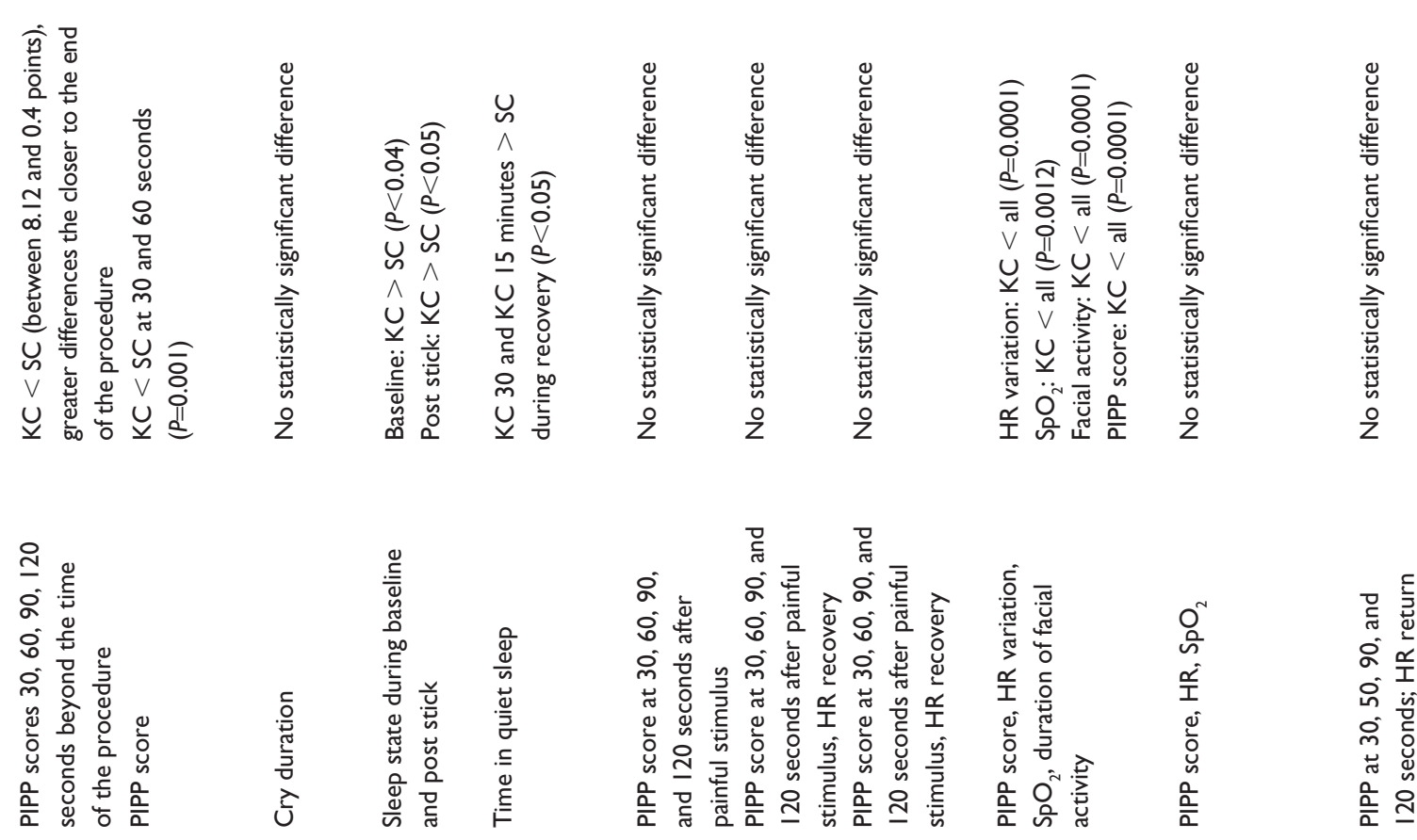

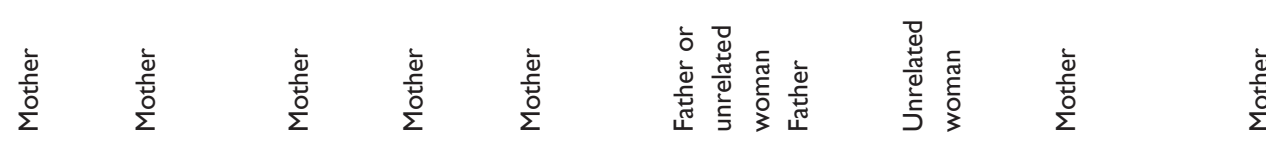

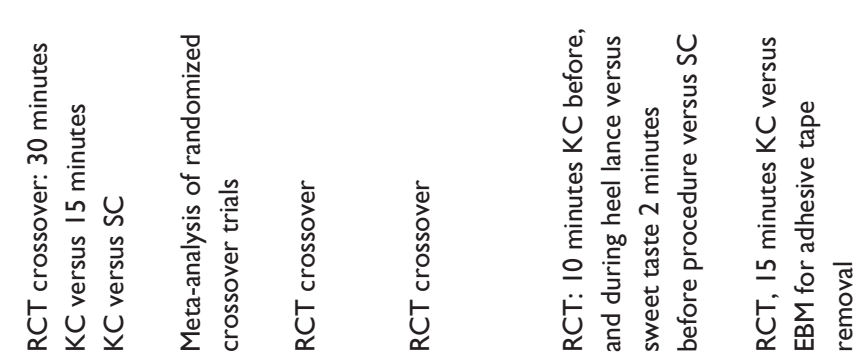

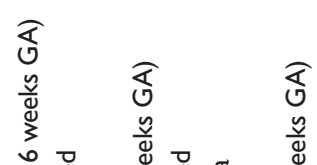
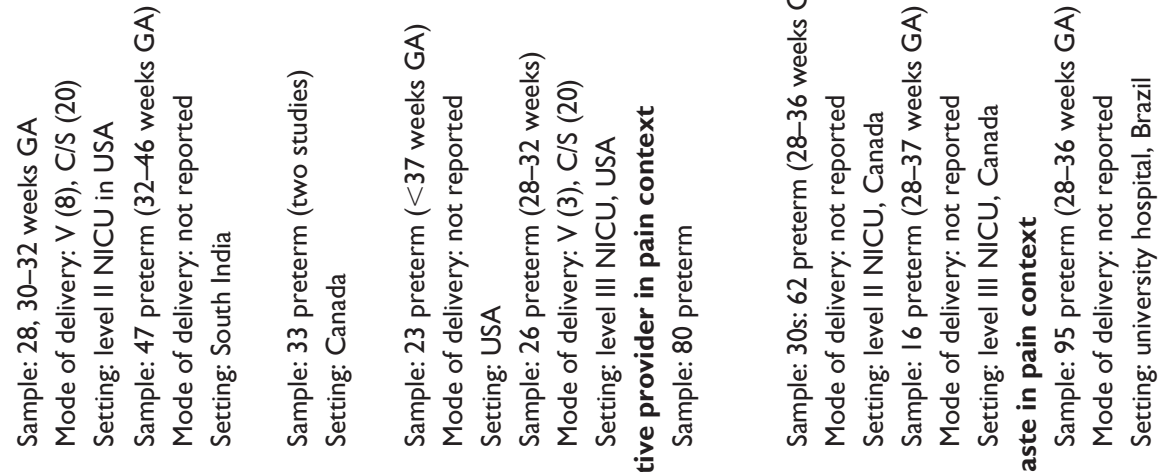

宛

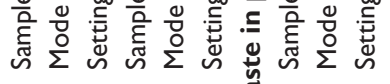
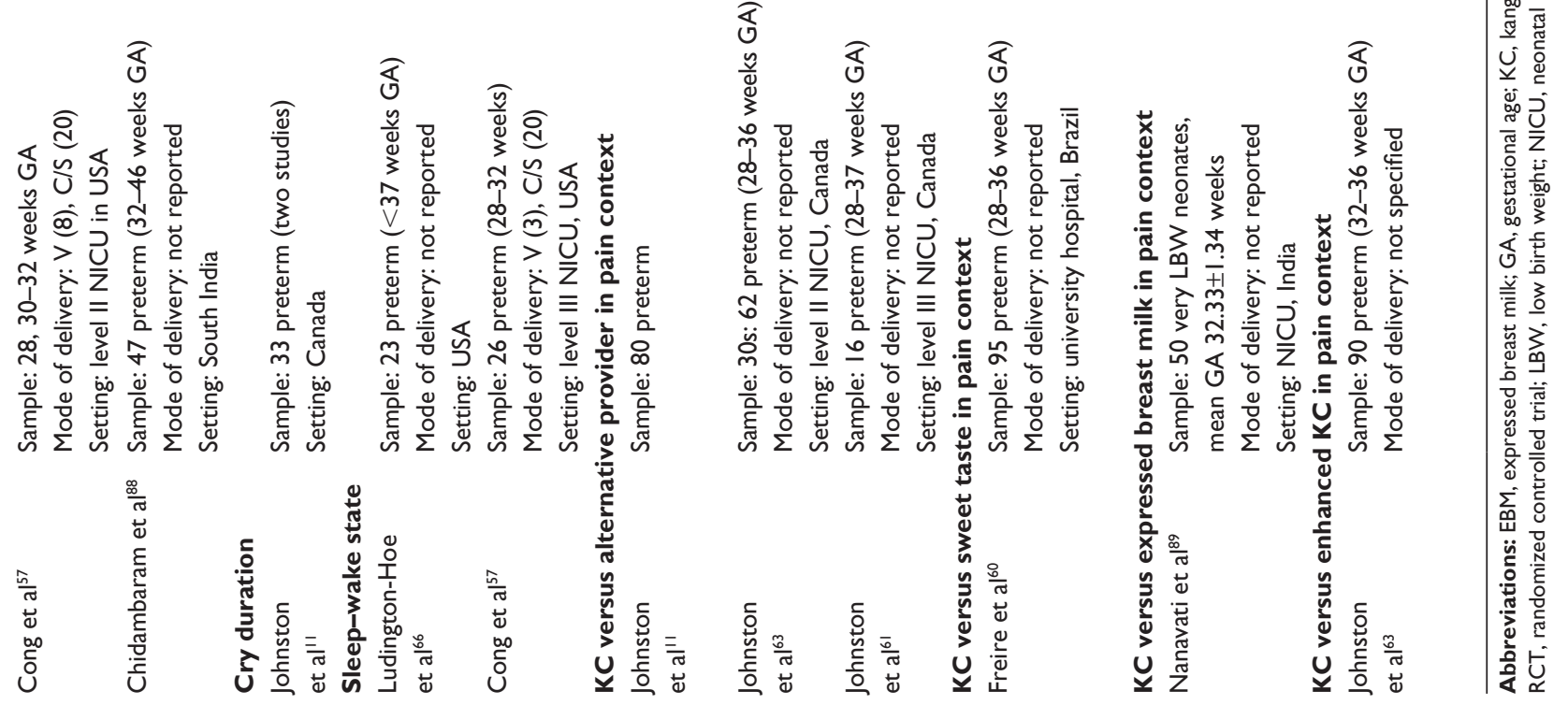
and/or heart rate recovery following a heel lance procedure. ${ }^{53,54,56-58,60,64,66,67,69-71}$ All studies favored $\mathrm{KC}$ or found no difference, but only a few studies could be combined in meta-analysis. Oxygen saturation during and/or following the painful procedure, reported by four studies, ${ }^{64,66,69,70}$ was unable to be combined. Although two of the studies ${ }^{64,70}$ reported that average oxygen saturation was higher in the $\mathrm{KC}$ group during the painful procedure, only Sajedi et $\mathrm{al}^{70}$ showed these differences to be significant. Of two studies reporting change in oxygen saturation, neither found a difference between $\mathrm{KC}$ and standard care. ${ }^{60,67}$

\section{Validated pain assessment tools}

Validated pain scores, including the Premature Infant Pain Profile (PIPP), ${ }^{54,55,59,60,64}$ Neonatal Facial Coding System, ${ }^{56,68}$ and Neonatal Infant Pain Scale ${ }^{68-70}$ were measured in ten studies. Pain scores regardless of the tool used appeared to favor KC with lower Neonatal Facial Coding System scores during procedure, with a mean difference of 1.872 in favor of $\mathrm{KC}(P<0.001)$ in Castral et al. ${ }^{56}$ Both Sajedi et $\mathrm{al}^{70}$ and Saeidi et $\mathrm{l}^{69}$ reported Neonatal Infant Pain Scale scores that significantly favored KC. Five studies used the PIPP as the outcome for heel lance. ${ }^{54,55,59,60,64}$ Based on the combined analysis of four studies, ${ }^{54,59,60,64}$ there was a significant effect post heel lance in favor of $\mathrm{KC}$ at 30 seconds, at 60 seconds, ${ }^{54,59,64}$ and 90 seconds. ${ }^{54,59,64}$ No significant difference between $\mathrm{KC}$ and controls were noted in PIPP scores at 120 seconds. ${ }^{54,59,64}$ Additional outcomes, including endocrine response, cry duration, infant state, and adverse effects, were measured in a few small studies, with mixed or nonsignificant findings. ${ }^{59}$

\section{Kangaroo care versus alternative treatments or alternative providers}

Comparisons have been made in four studies with sweet taste, ${ }^{60,68}$ breastfeeding, ${ }^{71}$ enhanced $\mathrm{KC}$ (including the addition of rocking and singing with $\mathrm{KC}),{ }^{62}$ fathers, ${ }^{62}$ and unrelated females. ${ }^{61}$ When $\mathrm{KC}$ with sweet taste (glucose) was compared with control in preterm neonates undergoing heel lance, heart rate, oxygen saturation variability, and PIPP scores all were reported to significantly favor KC. Similarly, heart rate, oxygen saturation, Neonatal Facial Coding System, and duration of crying were not reported to be different between $\mathrm{KC}$ and breastfeeding, but both were better than swaddled control. ${ }^{60}$ The addition of auditory, vestibular, or gustatory factors with KC (rocking, singing, and offering the infant a finger or pacifier for sucking by the mother providing $\mathrm{KC}$ ) did not result in any differences when compared with maternal $\mathrm{KC}$ alone. Two studies, both using a crossover design, compared the analgesic effect of maternal $\mathrm{KC}$ to SSC provided by an alternate caregiver: one with fathers ${ }^{62}$ and the other with an unrelated woman. ${ }^{61}$ In both cases, the differences in heart rate recovery and PIPP scores were not significant in spite of the large mean difference in favor of the mother, due mostly to high variance.

\section{Clinical implications}

$\mathrm{KC}$ is a simple, natural, and cost-effective intervention to effectively diminish behavioral pain response in preterm infants. KC should be routinely offered to all infants undergoing needle-related procedures. Although there is some evidence that combining $\mathrm{KC}$ provided by the mother with sweet-tasting solutions may be synergistic, further study is warranted before this combination can be recommended as standard care. In the absence of a mother, a father, ${ }^{62}$ unrelated woman, ${ }^{61}$ or a co twin, ${ }^{72,73}$ may be considered as as there is some evidence that they may be an effective alternate.

\section{Implications for research}

Despite strong evidence that $\mathrm{KC}$ effectively lowers composite behavioral pain scores for both full-term and preterm infants as young as 28 weeks' gestational age undergoing a single heel lance or intramuscular injection compared with incubator control, there remain many unanswered questions. Studies examining the sustained effect of $\mathrm{KC}$ over repeated procedures, as well as studies with larger sample sizes replicating prior work, including similar outcomes, are required. Moreover, little is known about whether combining $\mathrm{KC}$ with other comforting interventions can enhance its benefits for pain relief. It is clinically important for future studies to examine the optimal dose or duration of $\mathrm{KC}$ needed to be effective. The range of time for $\mathrm{KC}$ prior to the painful procedure was 2 minutes ${ }^{68}$ to 3 hours. ${ }^{66}$ The only studies that compared times were two studies by Cong et al. ${ }^{58,59}$ Although both studies seemed to favor 30 minutes to either longer ( 80 minutes $)^{58}$ or shorter doses (15 minutes) ${ }^{59}$ other studies using different outcomes favored $\mathrm{KC}$ for times longer and shorter than 30 minutes, so no conclusion can be made. Investigators should consider reporting findings from the entire sample as well as differentiating among gestational ages when possible. Lastly, examining whether the benefits of $\mathrm{KC}$ associated with early pain reduction and immediate pain relief may also lead to improved longer term outcomes are needed. 


\section{Considerations for implementation}

In spite of the plethora of documented benefits of $\mathrm{KC}$ in preterm infants, $\mathrm{KC}$ is not consistently practiced in this population. In a national survey of nurse managers in USA Newborn Intensive Care Units (NICUs), Engler et al ${ }^{74}$ found that while $82 \%$ of respondents indicated that $\mathrm{KC}$ was implemented in some form on their unit, practice was informed by nurse perceptions as opposed to scientific evidence. It has been consistently demonstrated that both parents and clinicians perceive $\mathrm{KC}$ as a positive intervention for mothers and their infants. ${ }^{75-79}$ However, despite generally positive attitudes, inconsistencies may exist in parent and nurse perceptions of optimal KC practices. For example, in a recent prospective cohort study conducted by Hendricks-Munoz et al, ${ }^{80}$ parents and nurses both reported that $\mathrm{KC}$ benefits infants; however, only $18 \%$ of nurses compared with $63 \%$ of mothers believed that $\mathrm{KC}$ should be provided to their infants on a daily basis.

In addition to varying attitudes toward $\mathrm{KC}$, numerous barriers have been identified. A consistently described barrier relates to the safety of facilitating $\mathrm{KC}$ in the preterm infant. Specifically, the highly technological equipment used to care for these infants has been identified as limiting opportunities for $\mathrm{KC},{ }^{74,76-79,81,82}$ with several studies reporting inconsistent policies and $\mathrm{KC}$ practices in infants who are intubated or have arterial or venous lines in place. ${ }^{74,81}$ Maternal concern around infant well-being during $\mathrm{KC}$, such as fear that the infant may stop breathing, is another concern that has been reported. ${ }^{77,79}$ Inadequate staff education and experience in facilitating $\mathrm{KC}$ for clinically compromised infants was another barrier identified, as well as lack of staff and time to appropriately support $\mathrm{KC}$ in both the NICU ${ }^{74,77,79,81}$ and following delivery by cesarean section. ${ }^{83}$ Both parents and health care providers identified the NICU environment as limiting parental visitation and opportunities to provide KC. For example, frequently identified barriers included lack of privacy, space, and comfortable chairs at the bedside, as well as a lack of facilities for meal preparation and parental rooming in. ${ }^{77,78,81,84}$ Parent-related factors such as maternal pain (eg, breast, back, or incisional pain), ${ }^{77,85}$ having responsibilities in the home (eg, other children, chores), ${ }^{77,81,85}$ and limited knowledge of the benefits of $\mathrm{KC}$ were additional barriers that were identified to limit the availability and motivation of mothers to provide $\mathrm{KC}{ }^{81}$

While it is evident that there are numerous barriers to successful implementation of $\mathrm{KC}$, findings also highlight ways in which to facilitate this practice in preterm infants. Educational interventions have been consistently cited as necessary to train staff in the knowledge and skills needed to promote and support KC effectively. ${ }^{74,76,80,81,86}$ In a recent prospective cohort study examining the impact of a training program on $\mathrm{KC}$ perceptions and practice competency, nurses received didactic education and simulation training for assessing and placing infants in KC. ${ }^{80}$ Nurses' competency in supporting $\mathrm{KC}$ for infants requiring nasal continuous positive airway pressure and mechanical ventilation improved from $30 \%$ to $92 \%(P<0.001)$ and from $10 \%$ to $48 \%(P<0.004)$, respectively. In addition, nurses who reported feeling uncomfortable in this competency decreased to $0 \%$, and the perceived value increased from $50 \%$ to $100 \%(P<0.001)$. Having clear policies in place to guide evidence-informed $\mathrm{KC}$ implementation, ${ }^{74,81,86}$ as well as KC leaders and unit-based champions, ${ }^{81}$ have also been identified as ways to facilitate ease of use in ill full-term and preterm infants.

In addition to educational interventions for staff, parent education regarding the benefits of $\mathrm{KC}$ and how to safely hold their infant in addition to assessing their well-behind has been documented as a way to encourage $\mathrm{KC}$ implementation. ${ }^{78,81,83,86,87}$ Modification of the physical environment by providing privacy screens, comfortable chairs, and family rooms has been identified by parents to support KC. ${ }^{77,78}$ Finally, assistance in positioning infants in $\mathrm{KC}$, providing parents with information and practical advice, as well as providing reminders and follow-ups around $\mathrm{KC}$ practices, ${ }^{77,78,81,87}$ have been identified by both parents and health care providers as valuable in supporting the implementation of $\mathrm{KC}$.

\section{Conclusion}

Kangaroo care is a natural, effective, and low-cost intervention that can be utilized in any setting. There is strong evidence related to its numerous benefits, including physiological, behavioral, and pain-relieving aspects for preterm newborns, both healthy and ill, as well as less stress and improved self-efficacy in parents. Mothers and family members have a unique relationship and are highly invested to ensure that optimal outcomes are achieved for their newborns. Yet their active participation in care often remains underutilized. Despite a few remaining unanswered questions, the use of $\mathrm{KC}$ should be considered standard of care for all infants and be initiated early with the ultimate goal to minimize separation of the mother-infant dyad.

More evidence is required in order to recommend implementation of $\mathrm{KC}$ in resource-rich environments. Though it is tempting to make this recommendation considering the diverse array of benefits offered, questions remain as to how implementation might affect mortality, infection, and severe illness. There a need for dose-response studies in this population that include a continuous or 
near-continuous arm, but these should be accompanied by economic measurements that will determine the tangible and intangible costs taken on by parents. To this end, health economists should be considered as potential members of the interdisciplinary team. Additionally, in poorly resourced countries, greater government funding should target the creation of $\mathrm{KC}$ centers that incorporate clean water, cooking, and sleeping facilities for mothers to remain exclusively with their infants.

\section{Disclosure}

The authors report no conflicts of interest in this work.

\section{References}

1. Konner M, Worthman C. Nursing frequency, gonadal function, and birth spacing among !Kung hunter-gatherers. Science. 1980;207(4432): 788-791.

2. Widstrom AM, Lilja G, Aaltomaa-Michalias P, Dahllof A, Lintula M, Nissen E. Newborn behaviour to locate the breast when skin-to-skin: a possible method for enabling early self-regulation. Acta Paediatr. 2011;100(1):79-85.

3. Charpak N, Ruiz JG, Zupan J, et al. Kangaroo mother care: 25 years after. Acta Paediatr. 2005;94(5):514-522.

4. Charpak N, de Calume ZF, Ruiz JG. The Bogota Declaration on Kangaroo Mother Care: Conclusions at the second international workshop on the method. Second International Workshop of Kangaroo Mother Care. Acta Paediatr. 2000;89(9):1137-1140.

5. Nyqvist KH, Anderson GC, Bergman N, et al. Towards universal kangaroo mother care: recommendations and report from the First European conference and Seventh International Workshop on Kangaroo Mother Care. Acta Paediatr. 2010;99(6):820-826.

6. Nyqvist KH, Anderson GC, Bergman N, et al. State of the art and recommendations. Kangaroo mother care: application in a high-tech environment. Acta Paediatr. 2010;99(6):812-819.

7. United Nations Children's Fund/World Health Organization. Babyfriendly hospital initiative. Geneva, Switzerland; United Nations Children's Fund/; 2009. Available from: http://www.unicef.org/nutrition/ files/BFHI_2009_s1.pdf. Accessed December 18, 2014.

8. Dordevic G, Jovanovic B, Dordevic M. An early contact with the babybenefit for the mother. Medicina Preglio. 2008;61(11-12):576-579.

9. Moore ER, Anderson GC, Bergman N, Dowswell T. Early skin-toskin contact for mothers and their healthy newborn infants. Cochrane Database Syst Rev. 2012;5:CD003519.

10. Conde-Agudelo A, Belizan JM, Diaz-Rossello J. Kangaroo mother care to reduce morbidity and mortality in low birthweight infants. Cochrane Database Syst Rev. 2014;3:CD002771.

11. Johnston C, Campbell-Yeo M, Fernandes A, Inglis D, Streiner D, Zee R. Skin-to-skin care for procedural pain in neonates. Cochrane Database Syst Rev. 2014;1:CD008435.

12. Christensson K, Siles C, Moreno L, et al. Temperature, metabolic adaptation and crying in healthy full-term newborns cared for skin-to-skin or in a cot. Acta Paediatr. 1992;81(6-7):488-493.

13. Ludington-Hoe SM, Nguyen N, Swinth JY, Satyshur RD. Kangaroo care compared to incubators in maintaining body warmth in preterm infants. Biol Res Nurs. 2000;2(1):60-73.

14. Legault M, Goulet C. Comparative study of two methods of holding premature infants: the kangaroo method versus the traditional method. Can J Nurs Res. 1993;24(4):67-80.

15. Chwo M, Anderson GC, Good M, Downling DA, Shiau SH, Chu D. A randomized controlled trial of early kangaroo care for preterm infants: effects on temperaure, weight, behaviour, and acuity. $J$ Nurs Res. 2002;10(2):129-141.
16. Marin Gabriel MA, Llana Martin I, Lopez Escobar A, Fernandez Villalba E, Romero Blanco I, Touza Pol P. Randomized controlled trial of early skin-to-skin contact: effects on the mother and the newborn. Acta Paediatr. 2010;99(11):1630-1634.

17. Bergman NJ, Linley LL, Fawcus SR. Randomized controlled trial of skin-to-skin contact from birth versus conventional incubator for physiological stabilization in 1200- to 2199-gram newborns. Acta Paediatr. 2004;93(6):779-785.

18. Ludington-Hoe SM, Anderson GC, Swinth JY, Thompson C, Hadeed AJ. Randomized controlled trial of kangaroo care: cardiorespiratory and thermal effects on healthy preterm infants. Neonatal Netw. 2004;23(3):39-48.

19. Mitchell AJ, Yates C, Williams K, Hall RW. Effects of daily kangaroo care on cardiorespiratory parameters in preterm infants. $J$ Neonatal Perinatal Med. 2013;6(3):243-249.

20. Rojas MA, Kaplan MD, Quevedo M, et al. Somatic Growth of Preterm Infants During Skin-to-Skin Care Versus Traditional Holding: A Randomized, Controlled Trial. Developmental and Behavioral Pediatrics. 2003;24(3):164-168.

21. Sloan NL, Camacho LW, Rojas EP, Stern C. Kangaroo mother method: randomised controlled trial of an alternative method of care for stabilised low-birthweight infants. Maternidad Isidro Ayora Study Team. Lancet. 1994;17(344):782-785.

22. Feldman R, Rosenthal Z, Eidelman AI. Maternal-preterm skin-toskin contact enhances child physiologic organization and cognitive control across the first 10 years of life. Biol Psychiatry. January 1, 2014;75(1):56-64.

23. Suman RP, Udani R, Nanvati R. Kangaroo mother care for low birth weight infants: a randomized controlled trial. Indian Pediatr. 2008;45(1):17-23.

24. Svensson KE, Velandia MI, Matthiesen AS, Welles-Nystrom BL, Widstrom AM. Effects of mother-infant skin-to-skin contact on severe latch-on problems in older infants: a randomized trial. Int Breastfeed J. 2013;8(1).

25. Chiu SH, Anderson GC. Effect of early skin-to-skin contact on motherpreterm infant interaction through 18 months: randomized controlled trial. Int. Nurs Stud. September 2009;46(9):1168-1180.

26. Tessier R, Charpak N, Giron M, Cristo M, de Calume ZF, Ruiz-Pelaez JG. Kangaroo Mother Care, home environment and father involvement in the first year of life: a randomized controlled study. Acta Paediatr. September 2009;98(9):1444-1450.

27. Nagai S, Andrianarimanana D, Rabesandratana N, Yonemoto N, Nakayama T, Mori R. Earlier versus later continuous Kangaroo Mother Care (KMC) for stable low-birth-weight infants: a randomized controlled trial. Acta Paediatr. June 2010;99(6):827-835.

28. Anderzen-Carlsson A, Lamy ZC, Eriksson M. Parental experiences of providing skin-to-skin care to their newborn infant- part 1: a qualitative systematic review. Int $J$ Qual Stud Health Well-being. 2014;13(9):24906.

29. Alfirevic Z, Milan SJ, Livio S. Caesarean section versus vaginal delivery for preterm birth in singletons. Cochrane Database Syst Rev. September 12, 2013.

30. Ludington-Hoe SM, Johnson MW, Morgan K, et al. Neurophysiologic assessment of neonatal sleep organization: preliminary results of a randomized, controlled trial of skin contact with preterm infants. Pediatrics. May 2006;117(5):e909-e923.

31. Scher MS, Ludington-Hoe S, Kaffashi F, Johnson MW, Holditch-Davis D, Loparo KA. Neurophysiologic assessment of brain maturation after an eight-week trial of skin-to-skin contact on preterm infants. Clin Neurophysiol. 2009;120(10):1812-1818.

32. Vohr BR. Neurodevelopmental outcomes of extremely preterm infants. Clin Perinatol. 2014;41(1):241-255.

33. Kugelman A, Colin AA. Late preterm infants: Near term bur still in a critical developmental time period. Pediatrics. 2013;132(4):741-751.

34. Kaffashi F, Scher MS, Ludington-Hoe SM, Loparo KA. An analysis of the kangaroo care intervention using neonatal EEG complexity: a preliminary study. Clin Neurophysiol. February 2013;124(2):238-246. 
35. Schneider C, Charpak N, Ruiz-Pelaez JG, Tessier R. Cerebral motor function in very premature-at-birth adolescents: a brain stimulation exploration of kangaroo mother care effects. Acta Paediatr. October 2012;101(10):1045-1053.

36. Alpanamayi B, Jagabandhu G, Arun KS, Avijit H, Suchandra M, Ranajit M. Effect of kangaroo mother care on growth and development of low-birthweight babies up to 12 months of age: a controlled clinical trial. Acta Paediatr. 2014(103):643-650

37. Nyqvist KH, Haggkvist AP, Hansen MN, et al. Expansion of the babyfriendly hospital initiative ten steps to successful breastfeeding into neonatal intensive care: expert group recommendations. J Hum Lact. August 2013;29(3):300-309.

38. Ahmed AH, Sands LP. Effect of pre- and postdischarge interventions on breastfeeding outcome and weight gain among premature infants. Journal of Obstetircal Gynecologic and Neonatal Nursing 2010;39(1):53-63.

39. Briere CE, Mcgrath J, Cong X, Cusson R. An integrative review of factors that influence breastfeeding duration for premature infants after NICU hospitalization. J Obstet Gynecol Neonatal Nurs. 2014;43(3):272-281.

40. Hurst NM, Valentine CJ, Renfro L, Burns P, Ferlic L. Skin-to-skin holding in the neonatal intensive care unit influences maternal milk volume. J Perinatol. 1997;17(3):213-217.

41. Flacking R, Ewald U, Wallin L. Positive effect of kangaroo mother care on long-term breastfeeding in very preterm infants. Joural of Obstetric, Gynecologic and Neonatal Nursing. 2011;40(2):190-197.

42. Heidarzadeh M, Bagher Hosseini M, Ershadmanesh M, Holamitabar Tabari M, Khazaee S. The effect of kangaroo mother care (KMC) on breast feeding at the time of NICU Discharge. Iran Red Crescent Med J. 2013;15(4):302-306.

43. Gathwala G, Singh B, Singh J. Effect of kangaroo mother care on physical growth breastfeeding and its acceptability. Trop Doct. 2010;40(4):199-202.

44. Hake-Brooks SJ, Anderson GC. Kangaroo care and breastfeeding of mother-preterm infant dyads 0-18 months: A randomized controlled trial. Neonatal Netw. 2008;27(3):151-159.

45. Donath SM, Amir LH, Team AS. Relationship between prenatal infant feeding intention and initiation and duration of breastfeeding: a cohort study. Acta Paediatr. 2003;92(3):352-356.

46. Flacking R, Lehtonen L, Thomson G, et al. Closeness and separation in neonatal intensive care. Acta Paediatr. October 2012;101(10):1032-1037.

47. Gathwala G, Singh B, Balhara B. KMC facilitates mother baby attachment in low birth weight infants. Indian J Pediatr. 2008 75(1):43-47.

48. Charpak N, Ruiz-Pelaez JG, Figueroa Z, Charpak Y. Kangaroo mother versus traditional care for newborn infants $</=2000$ grams: A randomized, controlled trial. Pediatrics. 1997;100(4):682-688.

49. Roberts KL, Paynter C, McEwan B. A comparison of kangaroo mother care and conventional cuddling care. Neonatal Netw. 2000;19(4):31-35

50. Neu M, Robinson J. Maternal holding of preterm infants during the early weeks after birth and dyad interaction at six months. J Obstet Gynecol Neonatal Nurs. July-August 2010;39(4):401-414.

51. Miles R, Cowan F, Glover V, Stevenson J, Modi N. A controlled trial of skin-to-skin contact in extremely preterm infants. Early Hum Dev. July 2006;82(7):447-455.

52. Lester BM, Hawes K, Abar B, et al. Single-family room care and neurobehavioural and medical outcomes in preterm infants. Pediatrics. 2014;134(4):754-760.

53. Gray L, Watt L, Blass EM. Skin-to-skin contact is analgesic in healthy newborns. Pediatrics. January 2000;105(1):e14.

54. Johnston CC, Stevens B, Pinelli J, et al. Kangaroo care is effective in diminishing pain response in preterm neonates. Arch Pediatr Adolesc Med. November 2003;157(11):1084-1088.

55. Akcan E, Yigit R, Atici A. The effect of kangaroo care on pain in premature infants during invasive procedures. Turk J Pediatr. JanuaryFebruary 2009;51(1):14-18.
56. Castral TC, Warnock F, Leite AM, Haas VJ, Scochi CG. The effects of skin-to-skin contact during acute pain in preterm newborns. Eur J Pain. May 2008;12(4):464-471.

57. Cong X, Cusson RM, Walsh S, Hussain N, Ludington-Hoe SM, Zhang D. Effects of skin-to-skin contact on autonomic pain responses in preterm infants. J Pain. July 2012;13(7):636-645.

58. Cong X, Ludington-Hoe SM, McCain G, Fu P. Kangaroo Care modifies preterm infant heart rate variability in response to heel stick pain: pilot study. Early Hum Dev. September 2009;85(9):561-567.

59. Cong X, Ludington-Hoe SM, Walsh S. Randomized crossover trial of kangaroo care to reduce biobehavioral pain responses in preterm infants: a pilot study. Biol Res Nurs. April 2011;13(2):204-216.

60. Freire NB, Garcia JB, Lamy ZC. Evaluation of analgesic effect of skinto-skin contact compared to oral glucose in preterm neonates. Pain. September 30 2008;139(1):28-33.

61. Johnston C, Byron J, Filion F, Campbell-Yeo M, Gibbins S, Ng E. Alternative female kangaroo care for procedural pain in preterm neonates: a pilot study. Acta Paediatr. November 2012;101(11):1147-1150.

62. Johnston CC, Campbell-Yeo M, Filion F. Paternal vs maternal kangaroo care for procedural pain in preterm neonates: a randomized crossover trial. Arch Pediatr Adolesc Med. September 2011;165(9): 792-796.

63. Johnston CC, Filion F, Campbell-Yeo M, et al. Enhanced kangaroo mother care for heel lance in preterm neonates: a crossover trial. J Perinatol. January 2009;29(1):51-56.

64. Johnston CC, Filion F, Campbell-Yeo M, et al. Kangaroo mother care diminishes pain from heel lance in very preterm neonates: a crossover trial. BMC Pediatr. 2008;8:13.

65. Kostandy RR, Ludington-Hoe SM, Cong X, et al. Kangaroo Care (skin contact) reduces crying response to pain in preterm neonates: pilot results. Pain Manag. Nurs June 2008;9(2):55-65.

66. Ludington-Hoe SM, Hosseini R, Torowicz DL. Skin-to-skin contact (Kangaroo Care) analgesia for preterm infant heel stick. AACN Clin Issues. July-September 2005;16(3):373-387.

67. Nimbalkar SM, Chaudhary NS, Gadhavi KV, Phatak A. Kangaroo Mother Care in reducing pain in preterm neonates on heel prick. Indian $J$ Pediatr. January 2013;80(1):6-10.

68. Chermont AG, Falcao LF, de Souza Silva EH, de Cassia Xavier Balda R, Guinsburg R. Skin-to-skin contact and/or oral 25\% dextrose for procedural pain relief for term newborn infants. Pediatrics. December 2009;124(6):e1101-e1107.

69. Saeidi R, Asnaashari Z, Amirnejad M, Esmaeili H, Robatsangi MG. Use of "kangaroo care" to alleviate the intensity of vaccination pain in newborns. Iran J Pediatr. March 2011;21(1):99-102.

70. Sajedi F, Kashaninia Z, Rahgozar M, Noghabi FA. The effect of Kangaroo Care on physiologic responses to pain of an intramuscular injection in neonates. Iranian Journal of Pediatrics. 2007;17(4): 339-344.

71. Okan F, Ozdil A, Bulbul A, Yapici Z, Nuhoglu A. Analgesic effects of skin-to-skin contact and breastfeeding in procedural pain in healthy term neonates. Ann Trop Paediatr. 2010;30(2):119-128.

72. Campbell-Yeo M, Johnston CC, Joseph KS, Feeley N., Chambers CT, Barrington $\mathrm{K}$. Co-bedding and time to recovery following heel lance in preterm twins [NCT00917631]. Pediatrics, 2012;130(3): 500-506.

73. Campbell-Yeo ML, Johnston CC, Joseph KS, et al. Co-bedding Between Preterm Twins Attenuates Stress Response After Heel Lance Results of a Randomized Trial. Clin J Pain. July 2014;30(7):598-604.

74. Engler AJ, Ludington-Hoe SM, Cusson RM, et al. Kangaroo care: national survey of practice, knowledge, barriers, and perceptions. $M C N$ Am J Matern Child Nurs. May-June 2002;27(3):146-153.

75. Ferrarello D, Hatfield L. Barriers to skin-to-skin care during the postpartum stay. MCN Am J Matern Child Nurs. January-February 2014;39(1):56-61.

76. Flynn A, Leahy-Warren P. Neonatal nurses' knowledge and beliefs regarding kangaroo care with preterm infants in an irish neonatal unit. J Neonatal Nurs. 2010;16(5):56-61. 
77. Blomqvist YT, Frolund L, Rubertsson C, Nyqvist KH. Provision of Kangaroo Mother Care: supportive factors and barriers perceived by parents. Scand J Caring Sci. June 2013;27(2):345-353.

78. Blomqvist YT, Nyqvist KH. Swedish mothers' experience of continuous Kangaroo Mother Care J Clin Nurs. May 2011;20(9-10): $1472-1480$.

79. Neu M. Kangaroo care: is it for everyone? Neonatal Netw. SeptemberOctober 2004;23(5):47-54.

80. Hendricks-Munoz KD, Li Y, Kim YS, Prendergast CC, Mayers R, Louie M. Maternal and neonatal nurse perceived value of kangaroo mother care and maternal care partnership in the neonatal intensive care unit. Am J Perinatol. November 2013;30(10):875-880.

81. Lee HC, Martin-Anderson S, Dudley RA. Clinician perspectives on barriers to and opportunities for skin-to-skin contact for premature infants in neonatal intensive care units. Breastfeed Med. April 2012;7(2):79-84.

82. Mallet I, Bomy H, Govaert N, et al. Skin to skin contact in neonatal care: knowledge and expectations of health professionals in 2 neonatal intensive care units. Arch Pediatr. July 2007;14(7):881-886.

83. Stevens J, Schmied V, Burns E, Dahlen H. Immediate or early skin-toskin contact after a caesarean section: A review of the literature. Matern Child Nutr. 2014:1-18.
84. Bergh AM, Manu R, Davy K, et al. Progress with the implementation of kangaroo mother care in four regions in Ghana. Ghana Med J. 2013;47(2):57-63.

85. Bazzano A, Hill Z, Tawiah-Agyemang C, Manu A, Ten Asbroek G, Kirkwood B. Introducing home based skin-to-skin care for low birth weight newborns: a pilot approach to education and counseling in Ghana. Glob Health Promot. September 2012;19(3):42-49.

86. Mangan S, Mosher S. Challenges to skin-to-skin kangaroo care: cesarean delivery and critically ill NICU patients. Neonatal Netw. July-August 2012;31(4):259-261.

87. Ahmed S, Mitra SN, Chowdhury AM, Camacho LL, Winikoff B, Sloan NL. Community Kangaroo Mother Care: implementation and potential for neonatal survival and health in very low-income settings. J Perinatol. May 2011;31(5):361-367.

88. Chidambaram AG, Manjula S, Adhisivam B, Vishnu BB. Effect of Kangaroo Mother Care in reducing pain due to heel prick among preterm neonates: a crossover trial. J Matern Fetal Neonatal Med. June $25,2014$.

89. Nanavati RN, Balan R, Kabra NS. Effect of kangaroo mother care vs expressed breast milk administration on pain associated with removal of adhesive tape in very low birth weight neonates: a randomized controlled trial. Indian Pediatr. November 8, 2013;50(11):1011-1015.

\section{Publish your work in this journal}

Pediatric Health, Medicine and Therapeutics is an international, peerreviewed, open access journal publishing original research, reports, editorials, reviews and commentaries. All aspects of health maintenance, preventative measures and disease treatment interventions are addressed within the journal. Practitioners from all disciplines are invited to submit

\section{Dovepress}

their work as well as healthcare researchers and patient support groups. The manuscript management system is completely online and includes a very quick and fair peer-review system. Visit http://www.dovepress.com/ testimonials.php to read real quotes from published authors. 